\title{
An Investigation of Wavelet Average Framing LPC for Noisy Speaker Identification Environment
}

\author{
Khaled Daqrouq, ${ }^{1}$ Rami Al-Hmouz, ${ }^{1}$ Abdullah Saeed Balamash, ${ }^{1}$ \\ Naif Alotaibi, ${ }^{1}$ and Elmar Noeth ${ }^{2}$ \\ ${ }^{1}$ Department of Electrical and Computer Engineering, Faculty of Engineering, King Abdulaziz University, Jeddah 21589, Saudi Arabia \\ ${ }^{2}$ Pattern Recognition Lab (Informatik 5), University of Erlangen-Nuremberg, Martensstrasse 3, 91058 Erlangen, Germany \\ Correspondence should be addressed to Rami Al-Hmouz; ralhmouz@kau.edu.sa
}

Received 15 March 2015; Revised 7 June 2015; Accepted 8 June 2015

Academic Editor: Eric Florentin

Copyright (C) 2015 Khaled Daqrouq et al. This is an open access article distributed under the Creative Commons Attribution License, which permits unrestricted use, distribution, and reproduction in any medium, provided the original work is properly cited.

In the presented research paper, an average framing linear prediction coding (AFLPC) method for a text-independent speaker identification system is studied. AFLPC was proposed in our previous work. Generally, linear prediction coding (LPC) has been used in numerous speech recognition tasks. Here, an investigative procedure was based on studying the AFLPC speaker recognition system in a noisy environment. In the stage of feature extraction, the speaker-specific resonances of the vocal tract were extracted using the AFLPC technique. In the phase of classification, a probabilistic neural network (PNN) and Bayesian classifier (BC) were applied for comparison. In the performed investigation, the quality of different wavelet transforms with AFLPC techniques was compared with each other. In addition, the capability analysis of the proposed system was examined for comparison with other systems suggested in the literature. In response to an achieved experimental result in a noisy environment, the PNN classifier could have a better performance with the fusion of wavelets and AFLPC as a feature extraction technique termed WFALPCF.

\section{Introduction}

Automatic speech recognition (ASR) is not a new problem to be studied. This subject has been tackled by many researchers, described in speech literature, and applied in several applications [1]. Now ASR is considered an essential tool for many applications, such as those used by people with hearing problems, and for voice-controlled services [2]. The heart of ASR is the speech feature extraction algorithm, and one of the most common algorithms used for feature extraction is based on the Karhunen-Loève transform (KLT). KLT has been successfully applied in speaker identification [3]. KLT is considered the optimal feature extraction technique for speech recognition applications in terms of the mean square error (MSE) and energy packing. Most speaker identification systems consider such features as the mel-frequency cepstral coefficient (MFCC) [4] and the linear predictive cepstral coefficient (LPCC) $[5,6]$. One of the weaknesses of MFCC is the use of short-time Fourier transform (SFT), which assumes that the signal is stationary and has weak time-frequency resolution. On the other hand, wavelet transform, which has good time-frequency resolution, is being widely used as a feature extraction method [7-9]. The wavelet transform is a decomposition method that helps in approximating the signal in hand using a set of wavelet coefficients. Such decomposition can be made at a different level of abstractions (scales). The way the signal is abstracted depends on the approximation function (the mother wavelet), which gives a wide range of possibilities for different applications. To have a coarser approximation of a signal, the mother wavelet is dilated and then translated over the signal time for approximation. Accordingly, a wavelet transform has two parameters: the scale and the translation parameters. The scale parameter can be a positive real number and the translation parameter can be an arbitrary real number; however, for computational efficiency, discrete values are often used [10,11]. Wavelet analysis has been used for feature extraction in speech recognition. In [12], the discrete wavelet transform (DWT) is used as a 
feature extraction method instead of discrete cosine transform. In [8], the wavelet transform is used to generate highenergy wavelet coefficients that suffer from shift variance, or subband energies are used instead of the mel filter-bank subband energies proposed in [13]. In [14], wavelet packet transform (WPT) bases are used to approximate the melfrequency division using Daubechies orthogonal filters. The difference between WPT and DWT is that WPT decomposes both details and approximations. It was found that feature extraction using WPT gave a better recognition performance compared to the DWT $[6,15]$. Nevertheless, the recognition time grows in a nonlinear fashion as a function of the number of wavelet packet bases. Therefore, the dimensionality of the problem becomes an issue. In [15], a feature extraction method based on a wavelet eigenfunction was proposed. In [9], a text-independent speaker identification system is proposed where the learning of the correlation between the wavelet transform and the expression vector is performed by the kernel canonical correlation analysis. WPT is used to perform the recursive. Defining the most relevant features is crucial in any learning problem, and speaker recognition is no exception $[16,17]$. One way to define the set of relevant features [6] is to use a criterion function that can show the classification power of the individual features. Sarikaya et al. [18] proposed a wavelet packet perceptual decomposition tree that yields the wavelet packet parameters (WPP). The authors in [19] proposed the energy indexes of DWT or WPT for speaker identification; WPT was superior in terms of recognition rate [6]. In [20], sure entropy was calculated for the waveforms of the terminal node signals obtained from DWT [20] for speaker identification. The Bayesian classifier (BC) classifies objects based on the statistical characteristics of the classes. It has been employed in many learning applications such as computer vision [21], text classification [22], target tracking [23], health diagnosis [24], and speech recognition $[6,25]$. A Bayesian classifier can utilize different features from different sources to produce a probabilistic classification model. Moreover, an optimal classifier is obtained as long as both the actual and estimated distributions agree on the most probable class. Bayesian classifiers give good performance even when strong feature dependencies are present [22, 23].

In this paper, the speaker identification system is studied in the context of recognition rate in noisy environments. This work studies several methods for improving previously published work [6]. Our purpose is to improve the performance of the AFLPC technique utility in several types of noisy environments. For this reason, many techniques, such as feature fusion with DWT or Shannon entropy, are investigated. The structure of this paper is as follows. We present the wavelet packet transform feature extraction method, followed by classification techniques. Next, results and discussion are presented. At the end of this paper, a conclusion is presented.

\section{The Wavelet Packet Transform Feature Extraction Method}

The wavelet packet could assist greatly in determining a quality feature extraction method for speaker recognition.
In the presented study, WPT is utilized for speaker feature extraction, but the resulted data has high dimensionality. Therefore, we need a better representation of speech features. In [26] authors presented the entropy value obtained from the wavelet norm in digital modulation recognition. In the biomedical field, [27] proposed a combination of the genetic algorithm based on a wavelet packet transform algorithm that was used in the pathological investigation, where the features were obtained by a group of wavelet packet coefficients. In [28] a robust speech recognition scheme for noisy environments was proposed by using the wavelet-based energy as a threshold for denoising estimation. In [19] the energy indexes of WP were proposed for a speaker identification task. For the speaker identification task, entropy was used for the waveforms obtained from DWT $[6,20]$. In [29] a feature extraction method for speaker recognition based on a combination of three entropy types (sure, logarithmic energy, and norm) was proposed. In this paper, we use LPCC that are obtained from WP tree nodes for speaker feature vector construction to be used for speaker identification. The proposed feature extraction method is summarized as follows.

(i) Silence removal and normalization: before the stage of features extraction, the speech data are processed by a silence removal algorithm followed by the application of a preprocessing, which is achieved by using the normalization of the speech signals. This produces speech signals with almost a closed maximum. Unequal signal amplitudes come from the different speaker volumes [6].

(ii) WP tree decomposition: speech signal is decomposed into WP at level three, and then the AFLPC is obtained from the WT subsignal:

$$
\left\{u_{q}(t)\right\}=\left\{u_{q 1}, u_{q 2}, \ldots, u_{q Z}\right\},
$$

where $Z$ is the number of considered frames (each frame is of $20 \mathrm{~ms}$ duration) for the $q$ th WT subsignal $u_{q}(t)$. The average of LPC coefficients calculated for $Z$ frames of $u_{q}(t)$ is utilized to extract a wavelet subsignal feature vector as follows:

$$
\operatorname{aflpc} c_{q}=\frac{1}{Z} \sum_{i=1}^{Z} \operatorname{LPC}\left(u_{q Z}\right) .
$$

The feature vector of the whole given speech signal is

$$
\mathrm{AFLPC}=\left\{\mathrm{aflpc}_{1}, \operatorname{aflpc}_{2}, \ldots, \operatorname{aflpc}_{\mathrm{Q}}\right\} .
$$

WP tree decomposition: speech signal is decomposed into WP at level seven, and we propose the Shannon entropy to extract features from each WP subsignal. The extracted features will be added to the AFLPC features for performancedeveloping investigation in the method proposed in [6]. To calculate Shannon entropy, the following equation is used:

$$
E(S)=-\sum_{i} S_{i}^{2} \log \left(S_{i}^{2}\right),
$$

where $S$ is the signal and $S_{i}$ are the WPT coefficients. 
In order to explain the proposed method in more detailed way the following chart is presented.

The dataset's speech signals are given to the silence removal and normalization block. In this block, speech signals are preprocessed to be ready for further feature extraction stages.

In the next stage, we decompose the signal into a WP tree that contains a number of WP's subsignals depending on the selected level. We may have 255 of WP's subsignals for a speech signal at level seven. Daubechies 5 wavelet function, denoted by ( $\mathrm{db} 5)$, is used.

Each subsignal, individually, is divided into $Z$ number of frames, and then we calculate 12 LPC coefficients for each frame. As the results of features from each subsignal, the average of the 12 LPC coefficient vectors is calculated over the $Z$ frames.

At this stage, we put the average of 12 LPC coefficients vectors obtained from all subsignals in one vector to form the feature vector of the speech signal.

At the end, Shannon wavelet entropy is calculated for each subsignal and added to the feature vector of the speech signal.

The validity of the proposed feature extraction method in the speaker identification task is shown in Figure 1 while Figure 1(a) illustrates two feature vectors of different speakers by the same method using the proposed method where they are less similar. Figure 1(b) illustrates two feature vectors of the same speaker taken for two different speech signals. It can be seen that the features have nearly similar shapes in Figure 1(b).

Linear prediction coding is one of the most attractive signals processing methods for speech signal analysis, particularly in speech and speaker recognition. In this work, LPCC with WP (LPCCWP) and AFLPC with WP (WPLPCF) were tested and compared for speaker recognition. The comparison was performed based on the recognition sensitivity (RS), which is proposed for the first time as follows:

$$
\mathrm{RS}=\left|\rho_{X X}-\rho_{X Y}\right|
$$

where $\rho_{X X}$ is the correlation coefficient calculated for the same speaker of different signals and $\rho_{X Y}$ is the correlation coefficient calculated for different speakers. The recognition sensitivity results calculated for 75 different signals show that there is a big difference between the two methods, where the LPCCWPF provides best results. The results are illustrated in Figure 2.

\section{Classification}

Several improvements, which include enhancements, extensions, and generalizations, have been proposed using a probabilistic neural network [30, 31]. The learning capability [32] and the classification accuracy of PNNs were the points of improvement [6]. This improvement will be reflected in the processing time [33] as well as the model complexity.

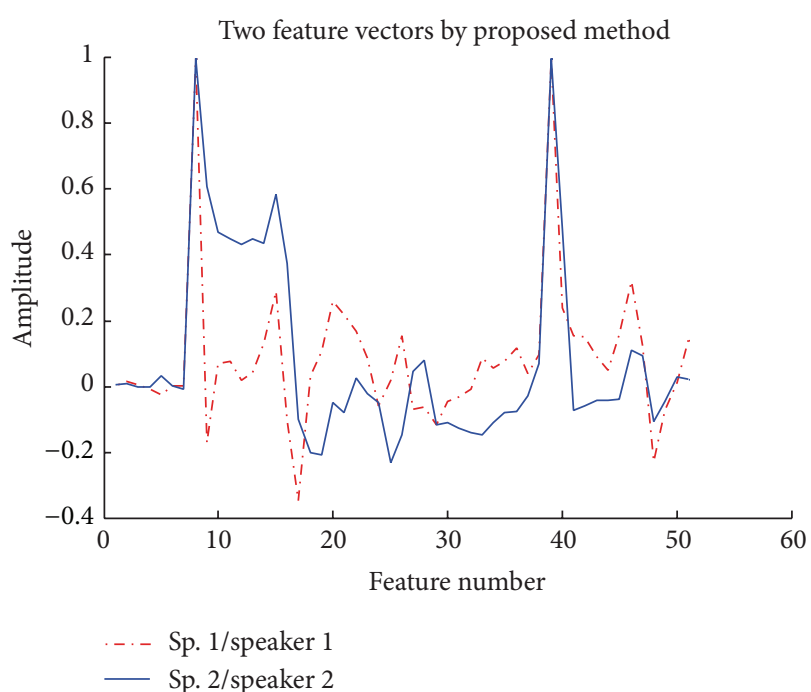

(a)

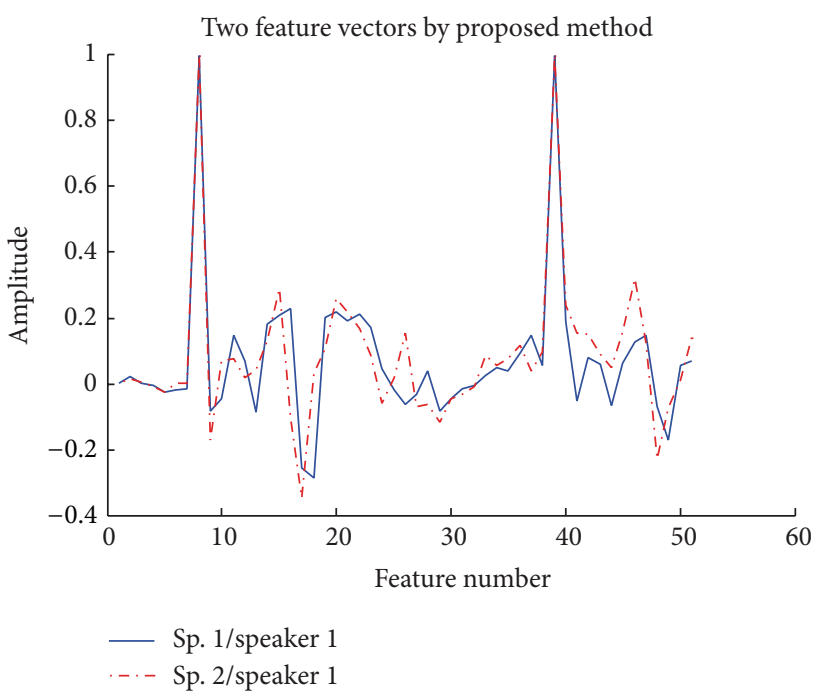

(b)

FIGURE 1: The proposed feature extraction method for two speech signals. (a) For two different speakers and (b) for the same speaker.

PNN is selected as the candidate classifier [6]. The characteristics of PNN to work in an unsupervised mode were the motivation for this selection. Also, PNN is easy to implement, and the output scores of the classes can be interpreted as the confidence (probability) for the class for the given feature at the input of PNN. The training phase of PNN is not time-consuming. So the implementation of PNN in many applications is considered because the training is achieved almost instantly. Though many improved types of the original probabilistic neural network exist, which are either more exhibited or significantly improved, for ease of explanation, we consider the original PNN for the classification. The suggested algorithm is indicated by PNN and depends on the 


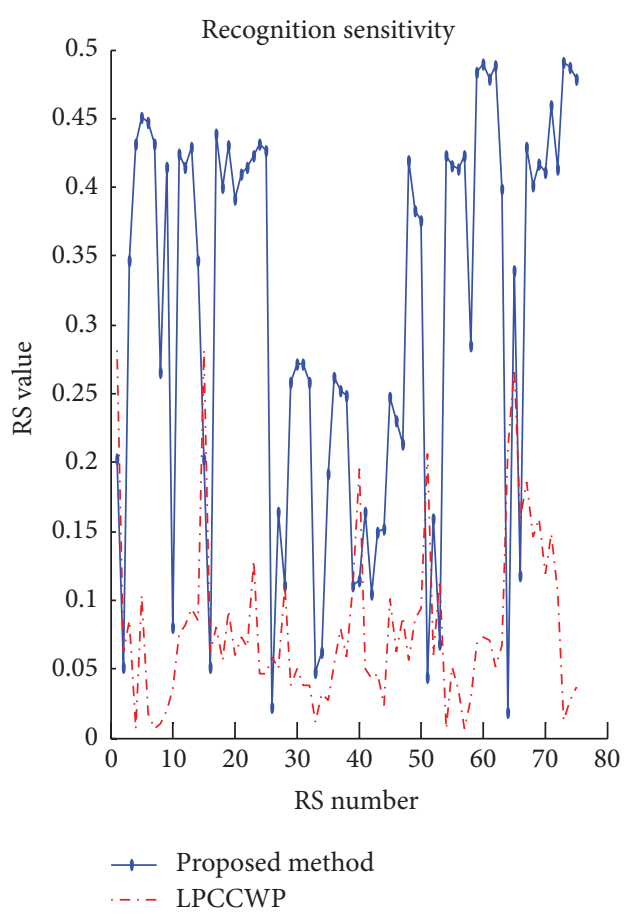

FIGURE 2: The recognition sensitivity calculated for proposed feature extraction method and for LPCC with WP (LPCCWP) at level 5.

following structure [6]. We have used a simple PNN in this work as follows:

$$
\text { Net }=\operatorname{PNN}(X, T, S P),
$$

where $X$ is a $180 \times 494$ matrix of input speaker feature vectors (pattern) of 180 average framing LPC coefficients, a method that was denoted above by AFLPC and taken from WP subsignals for net training. Information related to melfrequency cepstral coefficients and PNN that we used for comparison can be found in $[6,34]$. Consider

$$
X=\left[\begin{array}{cccc}
x_{1,1} & x_{1,2} & \cdots & x_{1,494} \\
x_{2,1} & x_{2,2} & \cdots & x_{2,494} \\
\vdots & \vdots & & \vdots \\
x_{180,1} & x_{180,2} & \cdots & x_{180,494}
\end{array}\right] .
$$

$T$ is the target class vector:

$$
T=[1,2,3,494] .
$$

The spread of the radial basis functions SP was set to the typical value ( $\mathrm{SP}=1)$. If the $\mathrm{SP}$ approaches zero, the network behaves as the nearest neighbor classifier. Meanwhile, if the SP goes higher, the designed network will take into account several nearby design vectors. We create two layer networks.

Naive Bayesian Classifier. Using these statistics of the features derived from AFLPC Method I (3), we can determine the likelihood of each feature belonging to a speaker to be verified. Let Class $C_{i}$ be a speaker class, and let $D$ be data that provides information (3) about $C_{i}$. Then $P\left(C_{i} \mid D\right)$ is calculated as follows:

$$
P\left(C_{i} \mid D\right)=\frac{P\left(D \mid C_{i}\right) P\left(C_{i}\right)}{\sum_{j} P\left(D \mid C_{j}\right) P\left(C_{j}\right)} .
$$

The first step is to estimate $P\left(D \mid C_{i}\right)$, usually referred to as the likelihood function. This is achieved using training speaker feature samples. Features are collected by applying DWT and DWTF on speaker signals. Figure 3 shows the histogram for three different speakers' features. It is clearly shown that using speaker feature statistics does not give abundant distinctions among speaker classes. Therefore, applying GMM [25] leads to low classification rates because speaker feature distributions, as in Figure 3, can be modeled as a single Gaussian distribution but with no discrimination among classes. Accordingly, we built a likelihood function for each feature (3) per speaker. It was found that most features can be modeled as a Gaussian distribution. For each feature, there would be a probability score for each speaker class. Therefore, let $f_{k}$ be a feature in the feature vector (3). Then $P\left(C_{i} \mid f_{k}\right)$ is calculated as follows

$$
\begin{gathered}
P\left(f_{k} \mid C_{i}\right)=\frac{P\left(f_{k} \mid C_{i}\right) P\left(C_{i}\right)}{\sum_{j} P\left(f_{k} \mid C_{j}\right) P(j)}, \\
P\left(C_{i} \mid f_{1} f_{1} \cdots f_{N}\right)=\frac{P\left(f_{1} f_{1} \cdots f_{N} \mid C_{i}\right) P\left(C_{i}\right)}{\sum_{j} P\left(f_{1} f_{1} \cdots f_{N} \mid C_{j}\right) P(j)} .
\end{gathered}
$$

$N$ is the total number of features in AFLPC (3). Under the assumption of conditional independence,

$$
P\left(C_{i} \mid f_{1} f_{1} \cdots f_{N}\right)=\frac{\prod_{k=1}^{N} P\left(f_{k} \mid C_{i}\right) P\left(C_{i}\right)}{\sum_{j} P\left(f_{1} f_{1} \cdots f_{N} \mid C_{j}\right) P(j)} .
$$

A posteriori $P\left(C_{i} \mid f_{1} f_{1} \cdots f_{N}\right)$ is computed throughout Bayesian fusion based on all features' probabilities in the speaker signal AFLPC. $P(C)$ is the prior probability of the speaker class $C_{i}$; it is assumed that all classes are equally likely $(P(C)=1 / 50) . \sum_{j} P\left(f_{1} f_{1} \cdots f_{N} \mid C_{j}\right) P(j)$ is a normalization term. The maximum a posteriori probability (MAP) of $C_{i}$ is used to estimate the speaker class $C_{i}$ that maximizes $P\left(C_{i}\right.$ | $\left.f_{1} f_{1} \cdots f_{N}\right)$ :

$$
\underset{i}{\operatorname{argmax}}\left\{P\left(C_{i} \mid f_{1} f_{1} \cdots f_{N}\right)\right\} .
$$

Similarly, features from different approaches can be combined to produce probability scores for each speaker. The essence of this approach is a way to combine different methods in a probabilistic manner. One method produces features that are different from other methods, and features from different methods suffer from independent noise. As a result of the combined methods, features will be more descriptive, and noise can be eliminated in the process of fusion.

\section{Results and Discussion}

The experimental setup was as follows. Speech signals were recorded via a PC sound card with a spectral frequency of 

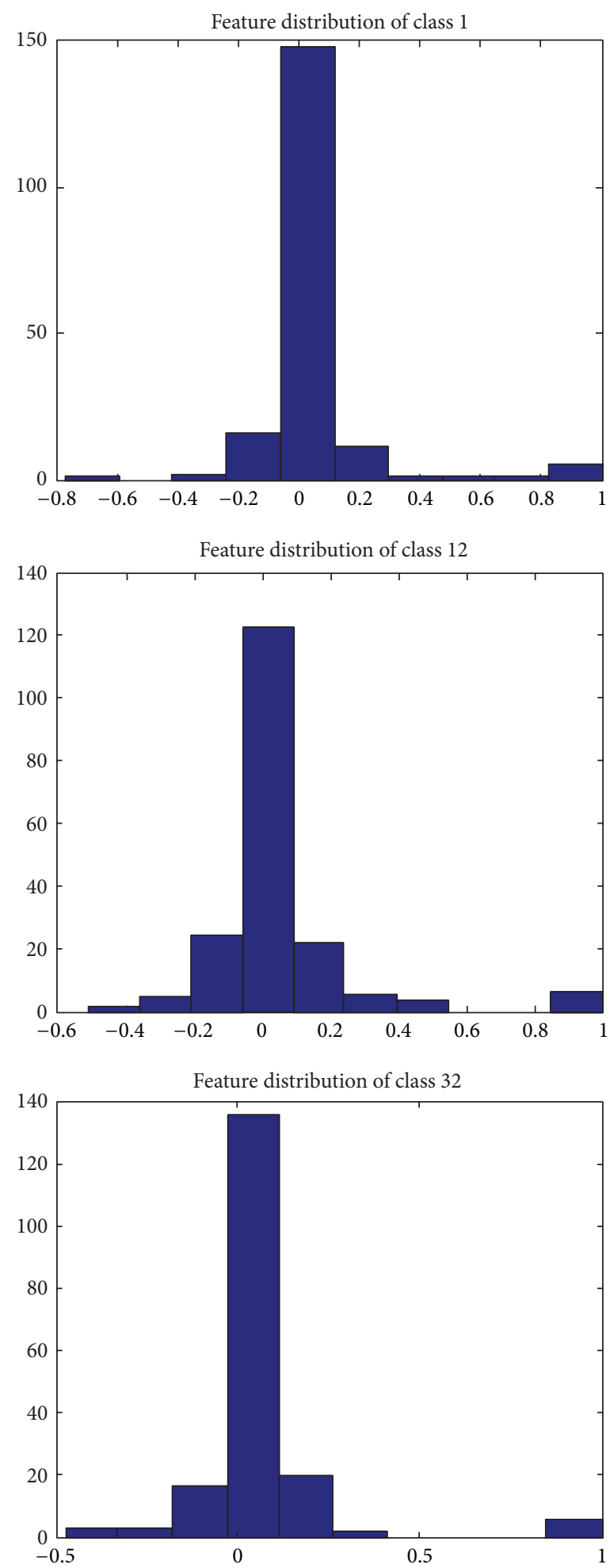

FIGURE 3: Speaker feature distributions.

$4000 \mathrm{~Hz}$ and a sampling frequency of $8000 \mathrm{~Hz}$. 50 people participated in the recordings. Each participant recorded a minimum of 20 different utterances in Arabic language. The age of the speakers varied from 20 to 45 years and included 28 males and 22 females. The recording process was performed in normal university office conditions. Our investigation of text-independent speaker identification system performance was performed via several experiments using 494 training signals and 50 classes. Even though speaker recognition performance has been maturing and improving over time, it 


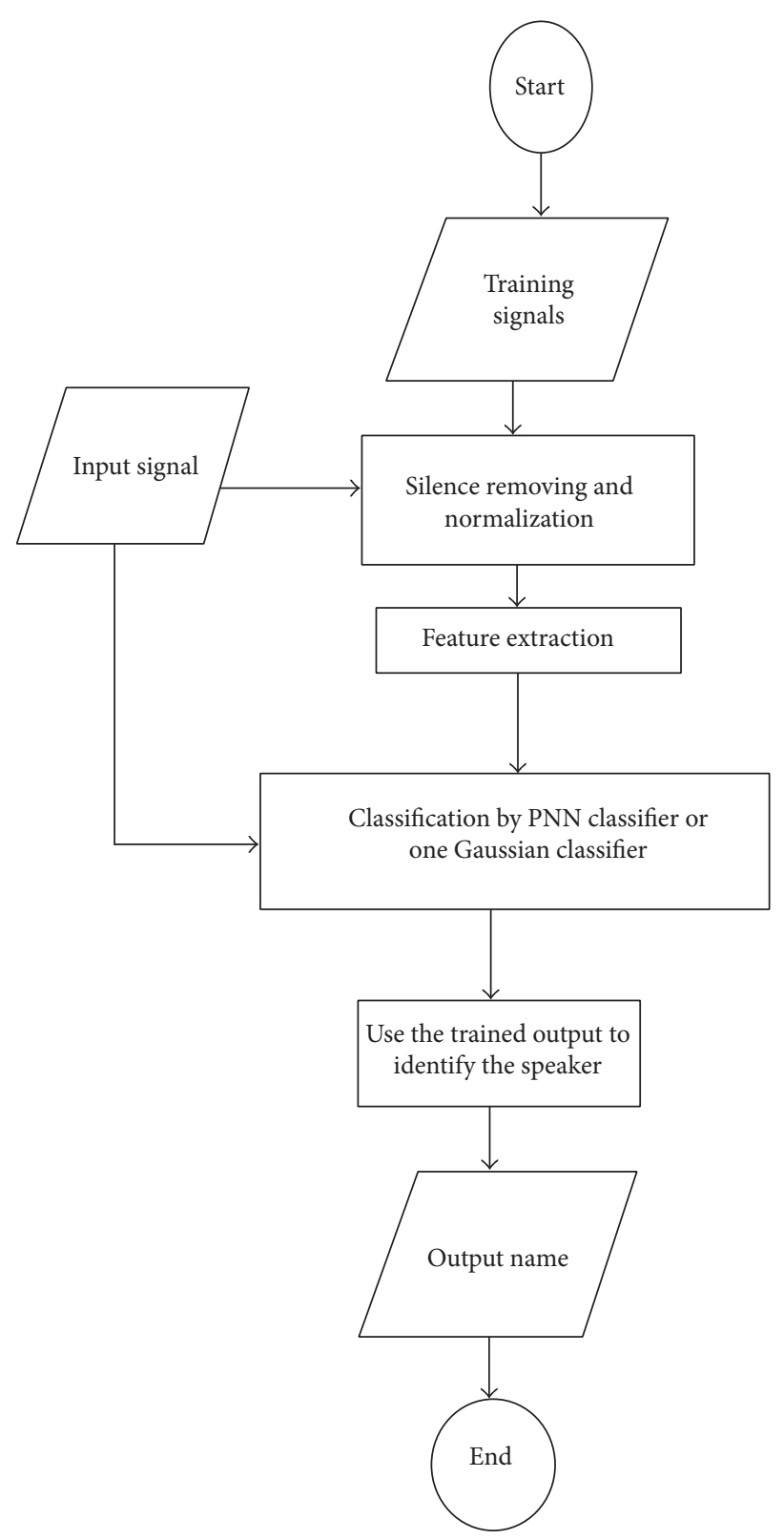

FIGURE 4: Flowchart for the proposed procedure.

is still inadequate in terms of accuracy [6]. In the approach, we propose a research study of speaker identification by wavelet transform in its two forms, DWT and WPT in noisy environments, which leads to the most comprehensive investigation. In other words, the presented study may be considered an investigation that aims to build a system that recognizes speakers even in a noisy environment. The system was applied to a huge number of training signals. We solved the problem by using the speaker recognition method (feature extraction and then classification). This approach was based on a combination of LPC and WT to accomplish feature extraction of the speakers obtained from normalized and silence-removed signals. The obtained feature extraction vector is added to the PNN or Bayesian classifier (BC) method to be classified, as seen in the flowchart presented in Figure 4.

Table 1 shows the results of the testing data of the whole database. Half of the signals from each individual speaker are used for training. Each test signal is classified by comparison with 494 trained signals by a classifier. The two classifiers are used for comparison. The results are compared to DWT with average framing LPC (DWLPCF) [12], DWT and WP fusion with AFLPC (WFALPCF), and WPLPCF and Shannon entropy obtained from WP at level 7 (WPLPCFE) [29]. The obtained results are tabulated in Table 1, where the best results were computed by means of WFALPCF and WPLPCF, $96.57 \%$ and $96.37 \%$, respectively, in case of PNN. BC showed excellent performances for DWT and WP fusion with AFLPC 
TABLE 1: Results of recognition rates (RR).

\begin{tabular}{lcccc}
\hline Method & WPLPCF & DWLPCF & WFALPCF & WPLPCFE \\
\hline RR\% (with PNN) & 95.77 & 73.19 & 96.57 & 96.37 \\
RR\% (with BC) & $24.32(94.15$ by WP2) & 90.14 & $33.23(96.57$ by WP2) & 20.21 \\
\hline
\end{tabular}

TABLE 2: Results of recognition rates for AWGN.

\begin{tabular}{|c|c|c|c|c|}
\hline Method & WPLPCF & DWLPCF & WFALPCF & WPLPCFE \\
\hline $\mathrm{RR} \%$ at $5 \mathrm{~dB}$ (with PNN) & 61.29 & 35.89 & 75.40 & 29.50 \\
\hline $\mathrm{RR} \%$ at $5 \mathrm{~dB}$ (with $\mathrm{BC}$ ) & 34.89 (61.49 by WP2) & 90.73 & 23.34 (61.57 by WP2) & 30.43 \\
\hline $\mathrm{RR} \%$ at $0 \mathrm{~dB}$ (with PNN) & 42.14 & 29.84 & 53.83 & 23.76 \\
\hline $\mathrm{RR} \%$ at $0 \mathrm{~dB}$ (with $\mathrm{BC}$ ) & 21.05 (51.59 by WP2) & 76.73 & 22.37 (34.13 by WP2) & 23.22 \\
\hline
\end{tabular}

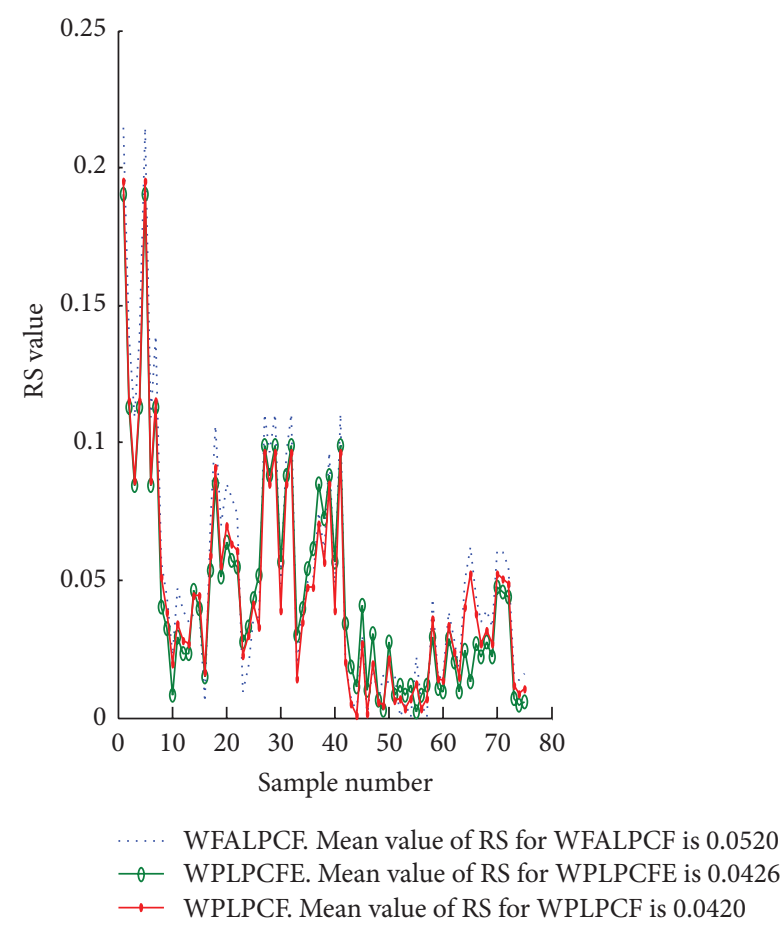

FIGURE 5: The recognition sensitivity calculated for the proposed feature extraction methods.

with WP at level 2 (WFALPCF WP2) with recognition rates reaching $96.57 \%$. BC failed in the WPLPCF of WP at the level 5 method. This happens because the longer the feature vector, the less the Gaussian distributed features.

In Figure 5, further investigation for a better feature extraction method is illustrated. WPLPCF, WFALPCF, and WPLPCFE were tested and compared for speaker recognition. The comparison was performed based on the recognition sensitivity (RS), which is proposed in Section 2 . The RS method is an objective way to evaluate the feature extraction method's ability to give similar feature vectors for the same speaker's signals and not similar ones for different speakers' signals. This gives a more elaborate study about the feature extraction methods. The recognition sensitivity results calculated for 75 different signals show that there is a slight difference between the presented methods, where the WFALPCF provides best results. The results are illustrated in Figure 5.

In the following experiments, several feature extraction methods with PNN and BC classifiers were analyzed to expose the usefulness of the proposed systems in noisy environments. The following experiment investigates the proposed method in terms of recognition rate in additive white Gaussian noise (AWGN) with 5 and $0 \mathrm{~dB}$. This can be concluded from interpretation of the results in Table 2, where the results of WPLPCF, DWLPCF, WFALPCF, and WPLPCFE are tabulated. DWT was processed at level 5 with six subsignals while WP was processed at level 5 with a bigger number of subsignals. It was found that the recognition rates (in case of $5 \mathrm{~dB}$ SNR) of DWLPCF at level 5 with BC were very good (90.73), but they failed in the case of using WP at level 5. WFALPCF methods were superior (75.40\%) in case of PNN. The same results were achieved in $0 \mathrm{~dB}$ SNR case.

The next experiment investigates the proposed method in terms of the recognition rate in Rayleigh distributed noise $(\mathrm{RDN})$, with 5 and $0 \mathrm{~dB}$. WPLPCF, DWLPCF, WFALPCF, and WPLPCFE were tested by means of PNN and BC. It was found that the WFALPCF methods were superior in 5 and $0 \mathrm{~dB}(90.52 \%$ and $84.27 \%)$ in the case of PNN. The results are tabulated in Table 3.

The next experiment investigated the proposed method in terms of recognition rate in babble real noise. The results are tabulated in Table 4, where WPLPCF, DWLPCF, WFALPCF, and WPLPCFE were tested by means of PNN and BC. It was found that the WFALPCF methods were superior in $5 \mathrm{~dB}(88.10 \%)$ in the case of PNN. In the case of $0 \mathrm{~dB}$, SNR WFALPCF with PNN (74.40\%) and DWLPCF with BC $(82.46 \%)$ were superior.

The last experiment investigated the proposed method in terms of recognition rate in subway real noise. The results are tabulated in Table 5. It was found that the WFALPCF methods were superior in $5 \mathrm{~dB}(89.11 \%)$ in the case of PNN. In the case of $0 \mathrm{~dB}$, SNR WFALPCF with PNN (75.12\%) and DWLPCF with BC (84.07\%) were superior.

At the end of our proposed method investigation study in noisy environments and by obtaining the average of 
TABLE 3: Results of recognition rates for Rayleigh noise.

\begin{tabular}{|c|c|c|c|c|}
\hline Method & WPLPCF & DWLPCF & WFALPCF & WPLPCFE \\
\hline $\mathrm{RR} \%$ at $5 \mathrm{~dB}$ (with PNN) & 61.70 & 53.83 & 90.52 & 31.44 \\
\hline $\mathrm{RR} \%$ at $5 \mathrm{~dB}$ (with $\mathrm{BC}$ ) & 27.54 (76.01 by WP2) & 84.89 & 22.21 ( 68.36 by WP2) & 26.44 \\
\hline $\mathrm{RR} \%$ at $0 \mathrm{~dB}$ (with PNN) & 79.03 & 45.77 & 84.27 & 33.67 \\
\hline $\mathrm{RR} \%$ at $0 \mathrm{~dB}$ (with BC) & 35.65 (69.76 by WP2) & 82.06 & 35.37 (54.23 by WP2) & 23.22 \\
\hline
\end{tabular}

TABLE 4: Results of recognition rates for babble noise.

\begin{tabular}{lcccc}
\hline Method & WPLPCF & DWLPCF & WFALPCF & WPLPCFE \\
\hline RR\% at $5 \mathrm{~dB}$ (with PNN) & 81.65 & 35.48 & 88.10 & 34.23 \\
RR\% at $5 \mathrm{~dB}$ (with BC) & $14.55(83.46$ by WP2) & 86.49 & $22.21(81.66$ by WP2) & 27.53 \\
RR\% at $0 \mathrm{~dB}$ (with PNN) & 66.33 & 32.48 & 74.40 & 21.53 \\
RR\% at $0 \mathrm{~dB}$ (with BC) & $12.02(67.54$ by WP2) & 82.46 & $14.84(48.19$ by WP2) & 19.12 \\
\hline
\end{tabular}

TABLE 5: Results of recognition rates for subway noise.

\begin{tabular}{lcccc}
\hline Method & WPLPCF & DWLPCF & WFALPCF & WPLPCFE \\
\hline RR\% at $5 \mathrm{~dB}$ (with PNN) & 80.04 & 44.56 & 89.11 & 32.10 \\
RR\% at $5 \mathrm{~dB}$ (with BC) & $30.02(81.67$ by WP2) & 87.50 & $30.30(87.30$ by WP2) \\
RR\% at $0 \mathrm{~dB}$ (with PNN) & 64.38 & 42.13 & 75.12 & 27.53 \\
RR\% at $0 \mathrm{~dB}$ (with BC) & $25.61(65.51$ by WP2) & 84.07 & $34.76(52.11$ by WP2) & 24.24 \\
\hline
\end{tabular}

TABLE 6: Results of average recognition rates for the four noise types.

\begin{tabular}{lc}
\hline Method & Average \\
\hline RR\% fusion with PNN & 78.84 \\
RR\% fusion with BC & 61.19 \\
RR\% with PNN & 56.96 \\
RR\% with BC & 49.90 \\
RR\% with WP & 63.13 \\
RR\% with DWT & 60.61 \\
\hline
\end{tabular}

recognition rates in terms of the following concepts (see Table 6), we can conclude that the best average result was achieved by means of WP and DWT fusion with PNN (78.84\%). The average result of the methods that used PNN gave the best result (61.19\%). According to wavelet transform, WP showed the best performance in the noise environment (63.13\%). The BC method gave excellent results in the case of the normal distribution of features as well as in the case of normal noise.

A standard dataset is an essential tool for testing the presented algorithms. The TIMIT database has been one of the most popular standard databases. This dataset contains 630 speakers of the same dialect. It contains 438 males and 192 females. For each speaker, ten signals with $8 \mathrm{KHz}$ sampling frequency were labeled (https://catalog.ldc.upenn.edu/LDC93S1). The same experiments of the four types of noise were conducted for the TIMIT database especially for the fusion methods with PNN and BC. 250 speakers were involved in the experiments. At each time, 50 speakers were used and averaged in the end to get the final result. Table 7 tabulates the results obtained for TIMIT.
TABLE 7: TIMIT results of average recognition rates for the four noise types.

\begin{tabular}{lc}
\hline Method & Average \\
\hline RR\% fusion with PNN & 61.31 \\
RR\% fusion with BC & 47.45 \\
\hline
\end{tabular}

\section{Conclusions}

In this work, the speaker identification task based on AFLPC was investigated in a noisy environment. The advantage of AFLPC is its ability to decrease the enormous speech data into a lower dimension for about $0.16 \%$ of the original size with a relatively high computing speed. For classification, $\mathrm{PNN}$ and $\mathrm{BC}$ were studied. We demonstrated the recognition rate of this method on a speaker database of 50 individual speakers (28 male speakers and 22 female speakers). 495 different signals were used for training the 50 speakers in the experiments. Investigational outcomes proved that both DWT and WP, linked with AFLPC (WFALPCF), are appropriate feature extraction techniques in noisy environments. However, in the case of PNN, DWT produced better quality than WP in terms of recognition rate with $\mathrm{BC}$. The results were achieved by bearing in mind the same feature vector length (180 coefficients, DWP at level 5, and WP at level 2 ). The results of the WP were enhanced by using higher levels, unlike DWT, which has no apparent enhancement after feature vector length extension. Compared with other available methods, WFALPCF achieved a higher recognition rate. 


\section{Conflict of Interests}

The authors declare that there is no conflict of interests regarding the publication of this paper.

\section{Acknowledgments}

This project was funded by the Deanship of Scientific Research (DSR), King Abdulaziz University, under Grant no. 12-135-35-RG. The authors, therefore, acknowledge with thanks DSR technical and financial support.

\section{References}

[1] J. Barker, N. Ma, A. Coy, and M. Cooke, "Speech fragment decoding techniques for simultaneous speaker identification and speech recognition," Computer Speech \& Language, vol. 24, no. 1, pp. 94-111, 2010.

[2] K. Daqrouq and T. A. Tutunji, "Speaker identification using vowels features through a combined method of formants, wavelets, and neural network classifiers," Applied Soft Computing, vol. 27, pp. 231-239, 2015.

[3] S.-Y. Lung and C.-C. T. Chen, "Further reduced form of Karhunen-Loeve transform for text independent speaker recognition," Electronics Letters, vol. 34, no. 14, pp. 1380-1382, 1998.

[4] D. Hosseinzadeh and S. Krishnan, "Combining vocal source and MFCC features for enhanced speaker recognition performance using GMMs," in Proceedings of the IEEE 9Th International Workshop on Multimedia Signal Processing (MMSP '07), pp. 365-368, Crete, Greece, October 2007.

[5] M. Afify and O. Siohan, "Comments on vocal tract length normalization equals linear transformation in cepstral space," IEEE Transactions on Audio, Speech and Language Processing, vol. 15, no. 5, pp. 1731-1732, 2007.

[6] K. Daqrouq and K. Y. Al Azzawi, "Average framing linear prediction coding with wavelet transform for text-independent speaker identification system," Computers \& Electrical Engineering, vol. 38, no. 6, pp. 1467-1479, 2012.

[7] O. Rioul and M. Vetterli, "Wavelets and signal processing," IEEE Signal Processing Magazine, vol. 8, no. 4, pp. 14-38, 1991.

[8] C. J. Long and S. Datta, "Wavelet based feature extraction for phoneme recognition," in Proceedings of the 4th International Conference on Spoken Language Processing (ICSLP '96), vol. 1, pp. 264-267, Philadelphia, Pa, USA, October 1996.

[9] S.-Y. Lung, "Improved wavelet feature extraction using kernel analysis for text independent speaker recognition," Digital Signal Processing, vol. 20, no. 5, pp. 1400-1407, 2010.

[10] S. Mallat and S. Zhong, "Characterization of signals from multiscale edges," IEEE Transactions on Pattern Analysis and Machine Intelligence, vol. 14, no. 7, pp. 710-732, 1992.

[11] S. Mallat, "Zero-crossing of a wavelet transform," IEEE Transactions on Information Theory, vol. 37, no. 4, pp. 1019-1033, 1991.

[12] Z. Tufekci and J. N. Gowdy, "Feature extraction using discrete wavelet transform for speech recognition," in Proceedings of the IEEE Southeastcon, pp. 116-123, IEEE, Nashville, Tenn, USA, April 2000.

[13] S. B. Davis and P. Mermelstein, "Comparison of parametric representations for monosyllabic word recognition in continuously spoken sentences," IEEE Transactions on Acoustics, Speech, and Signal Processing, vol. 28, no. 4, pp. 357-366, 1980.
[14] R. A. Mitchell, Hybrid Statistical Recognition Algorithm for Aircraft Identification, University of Dayton Press, Dayton, Ohio, USA, 1997.

[15] S.-Y. Lung, "Feature extracted from wavelet eigenfunction estimation for text-independent speaker recognition," Pattern Recognition, vol. 37, no. 7, pp. 1543-1544, 2004.

[16] C.-T. Chen, S.-Y. Lung, C.-F. Yang, and M.-C. Lee, "Speaker recognition based on 80/20 genetic algorithm," in Proceedings of the IASTED International Conference on Signal Processing, Pattern Recognition, and Application (SPPRA '02), pp. 547-549, Crete, Greece, June 2002.

[17] K. S. Nathan and H. F. Silverman, "Time-varying feature selection and classification of unvoiced stop consonants," IEEE Transactions on Speech and Audio Processing, vol. 2, no. 3, pp. 395-405, 1994.

[18] R. Sarikaya, B. Pellom, and J. Hansen, "Wavelet packet transform features with application to speaker identification," in Proceedings of the 3rd IEEE Nordic Signal Processing Symposium (NORSIG '98), 1998.

[19] J.-D. Wu and B.-F. Lin, "Speaker identification using discrete wavelet packet transform technique with irregular decomposition," Expert Systems with Applications, vol. 36, no. 2, pp. 31363143, 2009.

[20] D. Avci, "An expert system for speaker identification using adaptive wavelet sure entropy," Expert Systems with Applications, vol. 36, no. 3, pp. 6295-6300, 2009.

[21] R. Al-Hmouz and S. Challa, "License plate localization based on a probabilistic model," Machine Vision and Applications, vol. 21, no. 3, pp. 319-330, 2010.

[22] P. Domingos and M. Pazzani, "On the optimality of the simple Bayesian classifier under zero-one loss," Machine Learning, vol. 29, no. 2-3, pp. 103-130, 1997.

[23] B. Ristic, N. Gordon, and A. Bessell, "On target classification using kinematic data," Information Fusion, vol. 5, no. 1, pp. 1521, 2004.

[24] T. M. Mitchell, Machine Learning, McGraw-Hill, 1997.

[25] D. A. Reynolds, T. F. Quatieri, and R. B. Dunn, "Speaker verification using adapted Gaussian mixture models," Digital Signal Processing, vol. 10, no. 1-3, pp. 19-41, 2000.

[26] E. Avci, D. Hanbay, and A. Varol, "An expert discrete wavelet adaptive network based fuzzy inference system for digital modulation recognition," Expert Systems with Applications, vol. 33, no. 3, pp. 582-589, 2007.

[27] R. Behroozmand and F. Almasganj, "Optimal selection of wavelet-packet-based features using genetic algorithm in pathological assessment of patients' speech signal with unilateral vocal fold paralysis," Computers in Biology and Medicine, vol. 37, no. 4, pp. 474-485, 2007.

[28] R. Sarikaya and J. H. L. Hansen, "High resolution speech feature parametrization for monophone-based stressed speech recognition," IEEE Signal Processing Letters, vol. 7, no. 7, pp. 182$185,2000$.

[29] E. Avci, "A new optimum feature extraction and classification method for speaker recognition: GWPNN," Expert Systems with Applications, vol. 32, no. 2, pp. 485-498, 2007.

[30] D. F. Specht, "Probabilistic neural networks," Neural Networks, vol. 3, no. 1, pp. 109-118, 1990.

[31] W. Al-Sawalmeh, K. Daqrouq, O. Daoud, and A.-R. AlQawasmi, "Speaker identification system-based mel frequency and wavelet transform using neural network classifier," European Journal of Scientific Research, vol. 41, no. 4, pp. 515-525, 2010. 
[32] Z. R. Yang and S. Chen, "Robust maximum likelihood training of heteroscedastic probabilistic neural networks," Neural Networks, vol. 11, no. 4, pp. 739-747, 1998.

[33] D. F. Specht, "Enhancements to probabilistic neural networks," in Proceedings of the IEEE International Joint Conference on Neural Networks, Baltimore, Md, USA, June 1992.

[34] T. Ganchev, N. Fakotakis, and G. Kokkinakis, "Comparative evaluation of various MFCC implementations on the speaker verification task," Proceedings of the SPECOM, vol. 1, pp. 191-194, 2005. 


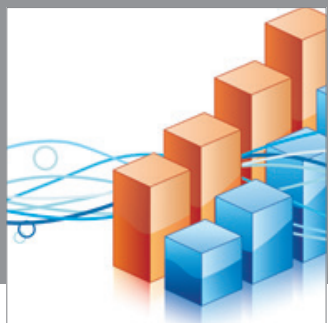

Advances in

Operations Research

mansans

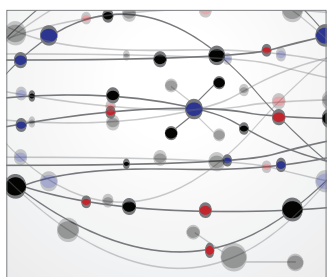

The Scientific World Journal
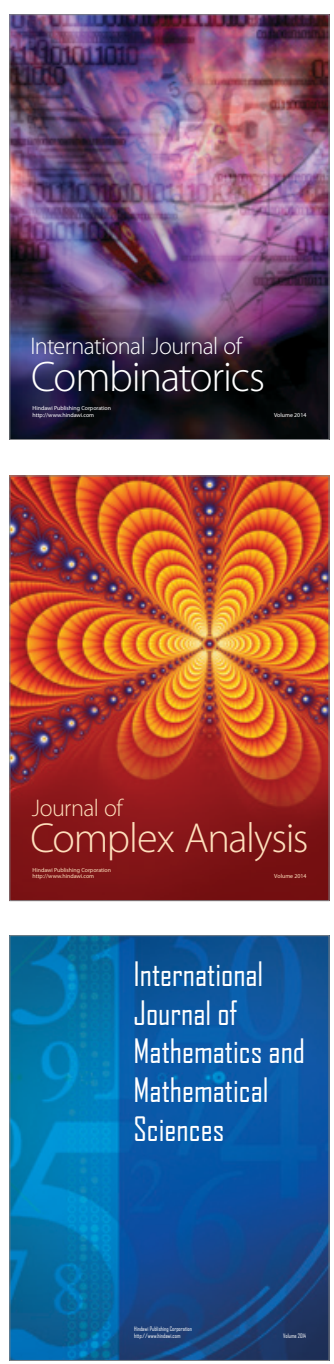
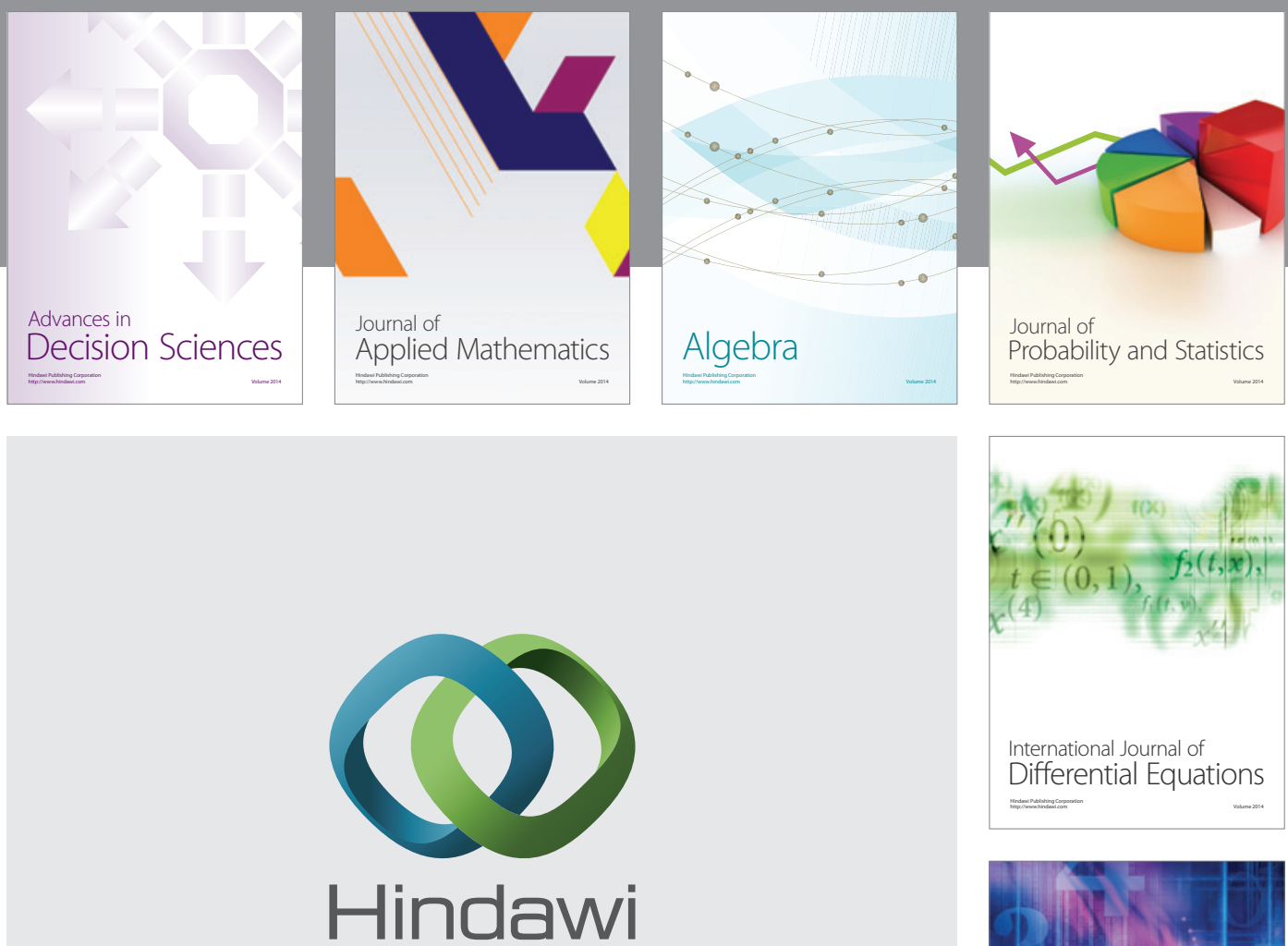

Submit your manuscripts at http://www.hindawi.com
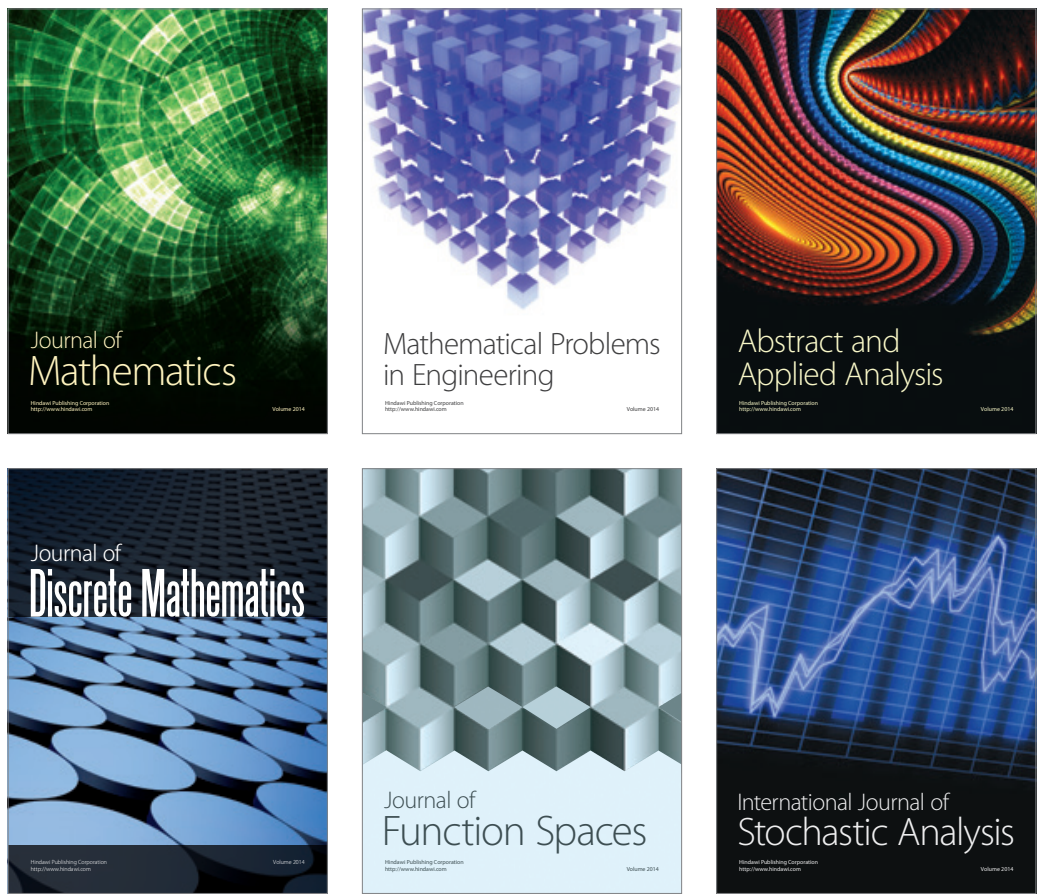

Journal of

Function Spaces

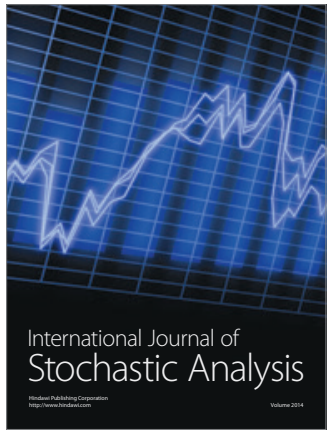

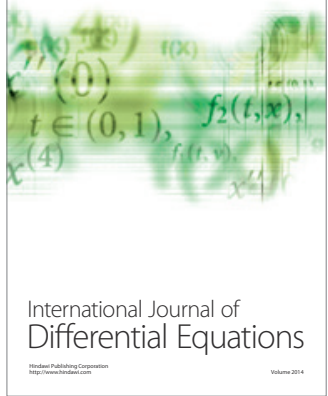
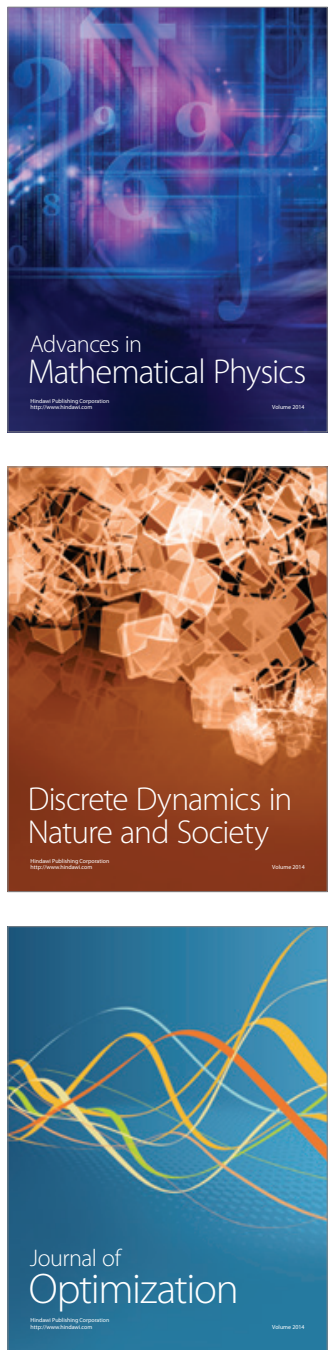